\title{
TOPOGRAFIA Y REACTIVIDAD DEL POTENCIAL EVOCADO VISUAL
}

\author{
R. ETCHEBERRIGARAY*-F. LOLAS* - S. CAMPOSANO* - D. ELGUETA **
}

\begin{abstract}
RESUMEN - El aumento - reducción (A-R) visual es evidente para las áreas centrales y exhibe diferencias hemisféricas. Este trabajo describe las características del potencial evocado visual (PEV) en derivaciones occipitales y su relación con A-R central: 16 sujetos de sexo masculino, diestros, jóvenes $y$ sanos recibieron flashes de $10 \mathrm{us}$, frec de $1 / \mathrm{s}$ a intensidades de $0.36, \quad 0.72$ y 1.44 joules mediante estimulador Grass PS2. Se registró derivaciones Cz, C3, C4, Oz, O1 y O2 referidas a mastoides mediante electrodos subdérmicos. Un computador Nicolet CA-1000 promedió 100 respuestas para cada intensidad. Se midió latencia y amplitud peak to peak de los complejos P1N1 y N1P2 y se calculó pendiente intensidad/amplitud mediante regresión lineal. 10 sujetos tuvieron pendiente mayor que 0 en vertex (aumentadores) y 6 menor (reductores). P1N1 presenta reducción y asimetría hemisferica en $\mathrm{Cz}$, que no se observa en Oz. Los aumentadores de N1P2 a nivel de $\mathrm{Cz}$ son reductores a nivel de Oz y viceversa. Se discuten estos hallazgos en relación a las etapas en el procesamiento de la información visual y la significación funcional de los componentes del PEV.
\end{abstract}

\section{Topography and reactivity of the visual evoked potencial.}

SUMMARY - Augmenting/reducing (A/R) of visual evoked potentials (VEP) has been repeatedly observed in central derivations, with some subjects increasing, and others decreasing, VEP amplitude with increasing intensity of stimulation. Central derivations also exhibit hemispheric lateralization regarding $\mathbf{A} / \mathbf{R}$. This paper explores central and occipital VEP in the same population of 16 healthy, right-handed male subjects, stimulated with binoculitr light flashes 10 usec in duration delivered by à Grass PS2 stimulator at a rate of 1/sec and at the intensities of $\mathbf{0 . 3 6}, 0.72$ and 1.44 joules at source. Amplitudes and latencies of components $\mathrm{P} 1, \mathrm{~N} 1$ and $\mathrm{P2}$ agreed with those reported in the literature. On the basis of amplitude/intensity slope functions of «peak to peak» amplitudes (P1N1 and NIP2) at Cz, 10 augmenters (slope greater than 0 ) and 6 reducers (slope smaller than 0 ) were found. Compared to central leads (C3 and C4) occipital ones (O1 and O2) did not exhibit significant interhemispheric differences. Vertex augmenters for N1P2 were occipital reducers and viceversa. The different characteristics of $A / R$ at occipital and central leads are interpreted in terms of stages of visual information processing in primary and association areas and functional significance of VEP components.

La amplitud de los componentes endógenos o tardíos del potencial evocado visual (PEV) no siempre aumenta paralelamente frente a incrementos en la intensidad de estimulación. Buchsbaum y Silverman 2,3 jdentificaron dos tipos de sujeitos en b. se a la reactividad electrocortical visual: aquellos que frente a incrementos de la int ansidađ de estimulación aumentan la magnitud del PEV, los "aumentadores" (Au) y los que presentan la respuesta opuesta, disminuyen la amplitud frente a aumentos en la intensidad de la estimulación, los "reductores" (Red). Estos resultados eran conc)rdantes con los trabajos sobre postefecto figural kinestésico (KFA) de Petrie 22, en que los sujetos estimaban el grosor de una barra graduada, dividiéndose tambien

* Unidad de Psicofisiologia, Depto. de Fisiología y Biofísica (DFB), Fac. de Medicina. Universidad de Chile; ** Ayudante-alumno del DF'B. 
en aumentadores y reductores, categorias que se relacionaban respectivamente con mayor o menor tolerancia al dolor; luego se postuló que el fenómeno aumento-reducción (A-R) reflejaría una propiedad general del sistema nervioso central, como mecanismo de regulación de la entrada sensorial. Buchsbaum $3,16,25$ relacionó el A-R al concepto pavloviano de "fuerza del sistema nervioso". Este concepto establece que el organismo responde linealmente frente al incremento de la intensidad de estimulación, hasta un determinado nivel - nivel de inhibición transmarginal - que sería un mecanismo de defensa, existiendo un punto en que el organismo comienza a disminuir la magnitud de la respuesta. De esta manera los sujetos con sistemá nervioso "débil" (reductores) presentan un umbral de "inhibición" más bajo que los con sistema nervioso fuerte (aumentadores). La postulación de un mecanismo general de A-R presenta algunos inconvenientes. En el potencial evocado auditivo (PEA) ha sido difícil evidenciar el fenómeno A-R; existen algunos datos en la literatura 21, y nuestro grupo ha presentado varios trabajos al respecto 7,8 . El problema central es la falta de correlación entre el A-R visual y el A-R auditivo. A este respecto son claros los trabajos de Kaskey y Raine 16,23. Afirman que la modalidad sensorial es un factor relevante al estudiar el A-R. Otro factor a considerar es la topografía de la respuesta electrocortical. Buchsbaum y col.2,3 observan reducción visual en la respuesta de vertex, no asi en areas occpitales. Estudios de nuestro grupo han mostrado diferencias hemisféricas del A-R en PEA y PEV. La evidencia actual indica, que en el PEA, el hemisferio izquierdo (HI) seria responsable del "aumento" y el hemisferio derecho (HD) de la "reducción". En el PEV se observa lo opuesto 4,6,20.

Estas diferencias sólo se observan al analizar la pendiente de la curva intensidad/amplitud, y no en el análisis aislado de las amplitudes. También hemos presentado evidencia preliminar acerca de diferencias en la reactividad y asimetria hemisférica de areas centrales y occipitales en el PEV 13,14.

El objetivo del presente trabajo es explorar la reactividad del PEV en areas centrales y occipitales, como forma de buscar diferencias en el procesamiento del estimulo visual en areas primarias (occipitales) y asociativas (centrales).

\section{MATERIATES Y METODO}

Sujetos - 16 voluntarios sanos, diestros, de sexo masculino, sin antecedentes mórbidos de importancia, ni consumo de alcohol, drogas o tabaco. Las edades fiuctuaron entre los 18 y 19 años. Estimulación - Consistió en flashes de 10 us de duración, con intervalo interestímulo de $1 \mathrm{~s}$ y 3 intensidades: $0.36,0.72$ y 1.14 joules. Fueron producidos por un estimulador fótico Grass PS2 y entregados mediante lámpara ubicada a $50 \mathrm{~cm}$ del sujeto. Se instruyó a éste a mantener la vista fija y evitar el pestaneo. Registro de PEV - Mediante electrodos subdérmicos con impedancias menores a 5000 ohms, se registró electroencefalograma. Se exploró derivaciones $\mathrm{Cz}, \mathrm{C} 3, \mathrm{C} 4, \mathrm{Oz}, \mathrm{O} 1 \mathrm{y} \mathrm{O} 2$, de acuerdo al sistema internacional 10-20 referidos a mastoides. La amplificación se efectuó con amplificador Nicolet HGA-200A, con sensibilidad $+-50 \mathrm{uV} / \mathrm{cm}$ y pasabanda de $1-100 \mathrm{~Hz}$. Un computador Nicolet CA-1000 promedió «on line» 100 respuestas para cada intensidad, con tiempo de análisis de 400ms. Mediante sistema de eliminación de artefactos se evitó la contaminación de actividad muscular, ocular y artefactos externos, como corriente alterna.

Protocolo experimental - Los registros fueron realizados en una sala con atenuación de Iuz y sonido. A los sujetos se les instaló en una silla cómoda instruyendolos a permanecer relajados con los ojos abiertos, evitando el pestaneo u otro movimiento. El registro se efectuó en bloques separados para las derivaciones centrales (Cz - Oz) y hemisféricas (C3, C4, O1, 02), debido a que el equipo dispone de 4 canales. El orden de presentación de las distintas intensidades fue aleatorio.

\section{RESULTADOS}

Las latencias de P1, N1, P2 estan dentro de rangos habituales; las amplitudes se refieren a mediciones «peak to peak». Las pendientes de la curva intensidad/amplitud se calcularon mediante regresión lineal. En la muestra total (Tabla 1) el complejo P1N1 tiene mayores amplitudes en hemisferio derecho (HD, C4) a intensidad de estimulación media $y$ máxima (Wilcoxon, $\mathrm{p}<0.05$ ). Las pendientes son positivas para $\mathrm{Cz}$ y $\mathrm{C4}$, en C3 es negativa $(4,5,8)$. En occipital no existen diferencias hemisféricas de amplitud, las pendientes son positivas en $\mathrm{Oz}$, O1 y $\mathrm{O} 2(14)$. N1P2 tiene mayores amplitudes en $\mathrm{C} 4$ que en C3. Las pendientes son negativas en $\mathrm{Cz}$ y $\mathrm{C3}$, en $\mathrm{C4}$ es positiva. A nivel occipital no hay diferencias en cuanto 


\begin{tabular}{|c|c|c|c|c|c|c|}
\hline P1N1 & $\mathrm{Cz}$ & $\mathrm{C} 3$ & $\mathrm{C} 4$ & $\mathrm{Oz}$ & 01 & $\mathrm{O} 2$ \\
\hline $\min 0.31$ & $6.6(3.0)$ & $7.2(3.5)$ & $7.1(5.2)$ & $7.7(3.5)$ & $5.1(2.6)$ & $5.5(2.2)$ \\
\hline med 0.72 & $7.6(3.9)$ & $6.5(2.7)$ & $* 8.6(4.9)$ & 10.0 & $5.8(3.2)$ & $7.3(4.0)$ \\
\hline $\max 1.44$ & $7.2(3.9)$ & $5.9(2.7)$ & * $9.1(5.8)$ & $10.0(6.6)$ & $6.7(4.3)$ & $7.9(4.4)$ \\
\hline pendiente & $0.6(3.7)$ & $-0.9(2.4)$ & $1.7(5.5)$ & $1.8(4.6)$ & $1.5(2.7)$ & $2.1(3.5)$ \\
\hline N1P2 & $\mathrm{Cz}$ & $\mathrm{C} 3$ & $\mathrm{C} 4$ & $\mathrm{Oz}$ & 01 & $\mathrm{O} 2$ \\
\hline $\min 0.34$ & $11.1(4.4)$ & $9.9(4.9)$ & $1.7(3.6)$ & $* * 4.2(2.4)$ & $3.4(1.9)$ & $4.0(2.7)$ \\
\hline med 0.72 & $10.9(3.8)$ & $9.4(5.0)$ & $10.8(5.1)$ & $* * 5.9(3.0)$ & $4.6(1.9)$ & 4.8 (2.1) \\
\hline $\max 1.44$ & $10.8(4.5)$ & 9.5 (3.9) & $* * 12.6(6.0)$ & $* * 4.5(3.0)$ & $4.6(2.2)$ & $5.0(2.4)$ \\
\hline pendiente & $-.28(3.7)$ & $-.26(2.4)$ & $* * .55(6.4)$ & $-.07\langle 2.9\}$ & $.04\langle 2.3\rangle$ & $.81(2.8)$ \\
\hline
\end{tabular}

Tabla 1 - Amplitudes $y$ pendientes de los complejos P1N1 y N1P2 en derivaciones centrales $y$ occipitales (Muestra total $N=16$ ). Wilcoxon: C3 is C4 y Cz vs Oz; $*$; 0.05 ; $* * p<0.01$.

a las amplitudes. La pendiente es negativa en $\mathrm{Oz}$, positiva en $\mathrm{O} 1 \mathrm{y}$ O2; y no hay diferencias interhemisfericas. Las amplitudes $y$ pendientes de P1N1 son mayores en $\mathrm{Oz}$ que en $\mathrm{Cz}$. Para N1P2 las amplitudes $y$ pendientes son mayores en $\mathrm{Cz}$ (ambas pendientes negativas, para todas las amplitudes $p<0.01$ ).

Aumento-Reducción en eI PEV - Se clasificó a los sujetos de acuerdo a la pendiente de la funcion intensidad/amplitud en el vertex (Cz) de los complejos P1N1 y N1P2 (i2); siendo los Aumentadores ( $\mathrm{Au}$ ) los que presentan pendiente positiva y Reductores (Red) los que tienen pendiente igual o menor a cero $(2)$.

Clasificando según P1N1 se encuentra 10 sujetos (Ss) aumentadores (62.5\%) y 6 reductores (37.5\%) (Tabla 2). Aumentadores: amplitudes y pendientes son mayores en C4 que en C3 para ambos complejos. A nivel occipital no hay diferencias interhemisféricas. Comparando Cz versus $\mathrm{Oz}$ en los Au, para P1N1 Oz presenta mayores amplitudes, para N1P2 Cz presenta mayores amplitudes $y$ pendientes (amplitud N1P2 intensidad mínima $p<0.01$, media y máxima $p<0.05$ ). Ređuctores: amplitudes media y máxima son mayores en C4 que c3, la pendiente es mayor en C4 $(\mathrm{p}<0.05)(4,20)$. A nivel occipital tampoco hay diferencia interhemisferica. Comparando $\mathrm{Cz}$ versus $\mathrm{Oz}$ en los Red, para $\mathrm{P} 1 \mathrm{~N} 1 \mathrm{Oz}$ presenta mayores amplitudes e intensidad media y maxima. La. pendiente es mayor en $\mathrm{Oz}(\mathrm{p}<0.05)$. Para $\mathrm{N}^{2} 2$ las amplitudes son mayores en $\mathrm{Cz}$ (minima $y$ media, $\mathrm{p}<0.05)$. La pendiente es mayor en $\mathrm{O} 7 \mathrm{p}<0.05)$.

\begin{tabular}{|c|c|c|c|c|}
\hline \multirow[t]{2}{*}{ Aumentadores } & \multicolumn{2}{|c|}{ P1N1 } & \multicolumn{2}{|c|}{ N1P2 } \\
\hline & $\mathrm{Cz}$ & $\mathrm{Oz}$ & $\mathrm{Cz}$ & $\mathrm{Oz}$ \\
\hline $\min$ & $5.4(3.3)$ & $7.6(3.4)$ & $9.9(3.9)$ & $* * 5.0(1.8)$ \\
\hline med & $7.0(3.7)$ & $9.6(4.7)$ & $10.4(4.2)$ & * 6.1 (3.2) \\
\hline $\max$ & $8.9(3.9)$ & $9.9(7.1)$ & $11.6(4.0)$ & $* 4.3(2.7)$ \\
\hline pendiente & $3.2(1.7)$ & $1.9(4.8)$ & $1.6(2.7)$ & $-.87(3.0)$ \\
\hline \multirow[t]{2}{*}{ Reductores } & \multicolumn{2}{|c|}{ P1N1 } & \multicolumn{2}{|c|}{ N1P2 } \\
\hline & $\mathrm{Cz}$ & $\mathrm{Oz}$ & $\mathrm{Cz}$ & $\mathrm{Oz}$ \\
\hline $\min$ & $7.9(1.5)$ & $7.8(3.7)$ & $13.1(4.6)$ & $* 3.0(2.7)$ \\
\hline med & $8.5(4.1)$ & $10.7(3.7)$ & $11.8(2.8)$ & * 5.5 (2.5) \\
\hline $\max$ & $4.3(1.8)$ & $10.1(5.5)$ & $9.4(5.0)$ & $4.7(3.5)$ \\
\hline pendiente & $-3.7(1.6)$ & * $1.7(4.2)$ & $-3.4 \quad(2.9)$ & $1.3(2.2)$ \\
\hline
\end{tabular}


Clasificando segun N1P2 se encuentra también 10 Au y 6 Red, 9 de los sujetos aumentadores en P1N1 también lo son para N1P2, en el grupo de los reductores 5 sujetos comparten la característica en los 2 complejos. Los análisis estadísticos arrojan los mismos resultados (Tabla 3). La pendiente de P1N1 sólo presenta reducción a nivel central, a nivel occipital es siempre positiva, incluso en el grupo de reductores a nivel de Cz. La pendiente de N1P2 presenta reducción en ambas regiones, pero siempre en sentido opuesto, los aumentadores en $\mathrm{Cz}$ presentan pendientes negativas en $\mathrm{Oz}$ y viceversa.

\begin{tabular}{|c|c|c|c|c|}
\hline \multirow[t]{2}{*}{ Aumentadores } & \multicolumn{2}{|c|}{ P1N1 } & \multicolumn{2}{|c|}{ N1P2 } \\
\hline & $\mathrm{Cz}$ & $\mathrm{Oz}$ & $\mathrm{C} z$ & $\mathrm{Oz}$ \\
\hline $\min$ & $5.7(3.5)$ & $8.1(2.9)$ & $10.3(4.5)$ & **: $4.3(1.3)$ \\
\hline med & $6.9(3.7)$ & $10.3(4.0)$ & $10.0(3.8)$ & * $5.4(2,7)$ \\
\hline $\max$ & $8.6(4.1)$ & $10.3(4.8)$ & $12.3(4.4)$ & $* 4.0(2.9)$ \\
\hline pendiente & $2.6(2.9)$ & $1.8(4.8)$ & 2.1 & $-.56(2.7)$ \\
\hline Reductores & \multicolumn{2}{|c|}{ P1N1 } & \multicolumn{2}{|c|}{ N1P2 } \\
\hline $\min$ & $\begin{array}{c}\mathrm{Cz} \\
7.4(1.4)\end{array}$ & $\begin{array}{c}\mathrm{Oz} \\
7.1^{(4.2)}\end{array}$ & $\begin{array}{c}\mathrm{Cz} \\
12.5(4.0)\end{array}$ & $\begin{array}{c}\mathrm{Oz} \\
* 4.1(3.5)\end{array}$ \\
\hline med & $8.6(4.1)$ & $9.5(5.0)$ & $12.4(3.2)$ & $* 6.6(3.2)$ \\
\hline $\max$ & $4.8(1.9)$ & $9.4(6.0)$ & $8.2(3.4)$ & $5.3(3.0)$ \\
\hline pendiente & $-2.8(2.0)$ & $* 1.9(4.2)$ & $-4.2(2.5)$ & $.74(3.1)$ \\
\hline
\end{tabular}

Tabla $3-A m p l i t u d e s$ y pendientes de P1N1 y N1PR en Cz y Oz (Aumentadores $N=10$, reductores $N=6$; critério $C z-N 1 P 2)$. Wilcoxon: Cz vs $0 z ; * p<0.05 ; * * p<0.01$.

\section{COMENTARIOS}

Los datos obtenidos del análisis del total de la muestra concuerdan con estudios previos e indican diferencias interhemisféricas del potencial evocado visual en regiones centrales, con predominio del HD. El HD presenta amplitudes y pendiente de la función intensidad amplitud mayores. Esto se observa en los dos complejos estudiados, y es compatible con la noción de un hemisferio derecho especializado en tareas de percepción visual global. En nuestra situación experimental no existe demanda para efectuar tareas analiticas o secuenciales, que pudieran implicar mayor actividad del hemisferio izquierdo. A nivel occipital sin embargo, no se observan diferencias interhemisféricas, la actividad de estas áreas, probablemente es el reflejo de una etapa precoz en el procesamiento de la información visual, que no presenta especialización hemisférica. Al efectuar la comparación $\mathrm{Cz}$ versus $\mathrm{Oz}$ se observan diferencias en el comportamiento de los complejos; el complejo P1N1 presenta mayores amplitudes y pendiente a nivel occipital, mientras que N1P2 es mayor en $\mathrm{Cz}$. Esto también apoya la idea de distintas etapas o diferentes procesos - en cuanto al análisis de la información visual - en areas occipitales y centrales. Es decir, el proceso precoz se efectuaría a nivel occipital, sin especialización hemisférica, reflejado principalmente por P1N1. A nivel central (areas asociativas) habría un proceso mas tardío y lateralizado, reflejado en el complejo N1P2.

Aumento/reducción - Independientemente del complejo analizado se encuentra la misma proporción de aumentadores y reductores, con predominio de los primeros (hallazgo habitual en estudios previos). Las diferencias interhemisféricas de reactividad a nivel central son: los aumentadores son más aumentadores en el HD y los reductores presentan mayor reducción en el HI. A nivel occipital no hay diferencias interhemisféricas para el complejo P1N1, en Au y Red. En N1P2 se observan diferencias interhemisféricas de amplitudes a nivel occipital, los Au tienen mayores amplitudes en $\mathrm{O} 1$ (HI), los Red en O2 (HD). Estas diferencias en el comportamiento de los complejos no tienen interpretación clara. La comparación central-occipital en Au y Red replica los hallazgos de la muestra global. Esta comparación aporta datos sobre el A-R a nivel occipital; el complejo P1N1 a nivel occipital presenta pendiente 
positiva tanto en Au como Red, en cambio N1P2, en $\mathrm{Oz}$ presenta pendiente positiva en los Red y negativa en los Au. Esto apoya la independencia funcional de los complejos.

En suma el PEV presenta diferencias claras en cuanto a su distribución en las distintas areas y en su reactividad. Ademas cada complejo presenta un comportamiento particular (como se ha descrito en detalle), probablemente por reflejar diferentes etapas $\mathrm{y} / \mathrm{o}$ procesos en el análisis de la informacion sensorial. También es posible especular que se trate de areas anatómicas distintas (primarias y asociativas), especializadas para cada etapa del procesamiento, con sus consiguientes diferencias electrofisiológicas.

Agradecimientos - Financiado por Depto. de Investigación y Bibliotecas, Univ. de Chile y FONDECYT. El primer autor tambiẹn agradece a la Fundación PUELMA.

\section{REFERENCIAS}

1. Birchall PMA, Claridge GS - Augmenting-reducing of the visual evoked potential as a function of changes in skin conductance level. Psychophysiology 16:482, 1979.

2. Buchsbaum $\mathbf{M}$, Haier $\mathbf{R}$, Johnson $\mathbf{J}-$ Augmenting and reducing: individual differences in evoked potentials. In Gale A, Edwards JA (eds): Physiological Correlates of Human Behavior, vol. 3. Academic Press, London, 1983.

3. Buchsbaum M, Silverman $J$ - Stimulus intensity control and the cortical evoked response. Psychosomatic Ned 30:12, 1968.

4. Collin C - Influencia de la especialización hemisférica y la modalidad sensorial sobre la reactividad cerebral. Tesis. Universidad de Chile. Santiago, 1985.

5. Collin C, Etcheberrigaray $R$, Lolas $F-$ Asimetria hemisférica de la reactividad cerebral: influencia de la modalidad sensoriai. Arch Biol Med Exper (Santiago) 17:R129, 1984.

6. Collin $C$, Etcheberrigaray $R$, Lolas $F-$ Hemispheric balance and sensory modality in vertex augmenting-reducing. IV World Congress of Biological Psychiatry. Abstracts. Philadelphia, 1985, pg 171.

7. Collin C, Lolas F - Hemispheric asymmetry of auditory evoked potential (AEP) augmenting-reducing. Psychophysiology 20:436, 1983.

8. Collin C, Lolas $\mathbf{F}$ - Hemispheric contribution to vertex augmentation/reduction of the auditory evoked potential. Arq Neuro-Psiquiat. (São Paulo) 43:347, 1985.

9. Collin C, Lolas $\mathrm{F}$ - Reactividad sensorial, asimetria hemisférica y diferencias individuales del comportamento. Rev Chil Psicol (Santiago) 6:9, 1983.

10. Davis AE, Wada JA - Hemispheric asymmetry: frequency analysis of visual and auditory evoked response to nonverbal stimuli, Electroencef Clin. Neurophysiol 37:1, 1974.

11. Dustman RE, Shaerer DE, Snyder EW - Age differences in augmenting-reducing of occipital visually evoked potentials. Electroenceph Clin Neurophysiol 54:99, 1982.

12. Etcheberrigaray $R$, Collin $C$, Lolas $F-$ Diferenciación psicofisiológica entre componentes tempranos y tardios del potencial evocado auditivo PEA). Psicopatología (Madrid) $6: 348,1986$.

13. Etcheberrigaray R, Elgueta D, Camposano S, Lolas F - Reactividad del potencial evocado visual (PEV) en areas centrales y occipitales. Primer Congreso Chileno de Ciencias Neurológicas y Psiquiátricas. Santiago, 1987. Resúmen 90 . 23, R90.

14. Etcheberrigaray $R$, Rees $R$, Camposano $S$, Lolas $F-$ Análisis topcgrafico del aumentoreducción (A-R) en el potencial evocado visua] (PEV). Rev Chil Neuropsiquiat (Santiago) $23: 4$ R14, 1985 .

15. Goodin DS, Waltz DA, Aminoff MJ - Task-dependent hemisphere asymmetries of the visual evoked potentlal. Neurology 35:378, 1985. 
16. Kaskey GB, Salzman LF, Klorman R, Pass $H L$ - Relationship between stimulus intensity and amplitude of visual and auditory event related potentials. Biol Psychol 10:115, 1980.

17. Knorring $L$ von, Monakhov $K$, Perris $C-$ Augmenting/reducing: an adaptative switch mechanism to cope with incoming signals in healthy subjects and psychiatric patients. Neuropsychobiology $4: 150,1978$.

18. Lolas F - Interhemispheric and sex difference in the visual evoked response recovery cycle. Neuropsychobiology 5:301, 1979.

19. Lolas F, Collin C, Camposano $\mathbf{S}$, Etcheberrigaray $\mathbf{R}$, Rees $\mathbf{R}$ - Hemispheric asymmetry of augmenting/reducing in visual and auditory evoked potentials. Biol Psychiat 22: $1413,1987$.

20. Lolas F, Garcia $\mathrm{H}$ - Potenciales cerebrales ligados a la estimulación visual. Acta Neurol Latinoamer $23: 155,1977$.

21. Orlebenke JF, Kok A, Zeillemaker CW - Augmenting-reducing (desinhibition) and the processing of auditory stimulus intensity: an ERP study. Psychophysiology 21:591, 1984 .

22. Petrie A, Collins W - Perceptual differences as related to the tolerance of pain suffering. Acta Psychol 19:755, 1961.

23. Raine A, Mitchell DA, Venables PH - Cortical augmenting-reducing: modality specific? Psychophysiology 18:700, 1981.

24. Rockstroh B, Thomas E, Birbaumer N, Lutzenberger W - Slow Brain Potentials and Behavior. Urban \& Schwarzenberg, Baltimore, 1982.

25. Silverman J, Buchsbaum M, Stierlin S - Sex differences in differenciation and stimulus intensity control. J Personal Soc Psychol 25:309, 1973.

26. Sobotka S, Pizlo Z, Budohoska W- Hemispheric differences in evoked potentials to pictures of faces in the left and the right visual field. Electroenceph Clin Neurophisiol $59: 441,1984$. 